\title{
Aproximación a un estado del arte en torno al constructivismo aplicado al derecho*
}

\author{
Approximation to a state of the art regarding constructivism applied to law
}

\begin{abstract}
Alejandro Pesca Salazar ${ }^{* *}$
*Artículo derivado de tesis de grado maestría en educación Aproximación a un estado del arte en torno al constructivismo aplicado al derecho, (2019), avalado por la Facultad de Educación de la Universidad Militar

Nueva Granada.

**Abogado y Magister en Educación, correo:serjuesItda2006@hotmail.com
\end{abstract}

Fecha de recepción: 15 junio de 2019

Fecha de aprobación: 30 noviembre de 2019

Para citar este artículo / To reference this article

Pesca, A. (2019) Aproximación a un estado del arte en torno al constructivismo aplicado al derecho. Inciso, 21(2);207-228.

Resumen

DOI: http://dx.doi.org/10.18634/incj.21v.2i.986

El presente artículo responde a la pregunta: ¿Cuáles son las investigaciones más relevantes que se han trabajado en términos del constructivismo aplicado al derecho? Para resolver esta pregunta se fija como objetivo realizar una investigación aproximada del estado del arte en torno a la aplicación del constructivismo en el campo del derecho. Por lo tanto, se tiene como impresión de sentido que la teoría constructivista jurídica ha sido estudiada por diferentes investigadores a nivel nacional e internacional, es así como nace la necesidad de realizar un estado del arte que recopile los textos elaborados en este sentido. La metodología utilizada para el desarrollo del objetivo fue interpretativa hermenéutica mediante la revisión de textos, publicaciones, ensayos, papers, artículos de revistas indexadas y demás textos de análisis documental. Como conclusión; se determinó que se realizó un rastreo de aportes en los enfoques constructivistas del derecho para que se pueda establecer un amplio y significativo referente bibliográfico.

Palabras clave: Análisis documental, constructivismo, derecho, epistemología, investigación. 


\section{ABSTRACT}

This article answers the question: What are the researches more relevant that have been worked on in terms of constructivism applied to the law? To solve this question, the objective is to carry out an approximate investigation of the state of the art around the application of constructivism in the field of law. Therefore, it is understood that the legal constructivist theory has been studied by different researchers at national and international level that is how the need to create a state of art that compiles the texts developed in this sense is born. The methodology used for the development of the objective was interpretative hermeneutics through the revision of texts, publications, essays, papers, articles of indexed journals and other texts of documentary analysis. Conclusion; It was determined that a tracking of contributions was carried out in the constructivist approaches of the law so that a broad and significant bibliographical reference could be established.

Keywords: constructivism, research, epistemology, legal, documentary análysis, criticism, higher education, state of the art, law. Constructivismo en el ámbito del derecho

\section{El constructivismo en el ámbito de la enseñanza del Derecho}

El constructivismo en el ámbito de la enseñanza del derecho, como enfoque educativo y/o formativo, es bastante novedoso y las investigaciones escritas, publicaciones y referencias bibliográficas, estados del arte son escasos hasta el momento, por ello, esta investigación está centrada en obtener a través de un barrido literario datos significativos que sean aporte para investigadores en este tema. En este documento el lector encontrará un estado del arte sobre las investigaciones y producciones escritas de autores nacionales e internacionales en cuanto se trata del constructivismo aplicado al derecho, esto con el fin de obtener suficientes referencias bibliográficas para información de los investigadores.

Este análisis es pertinente y se justifica porque da lugar al aporte crítico de los referentes bibliográficos y sus posiciones, basados en el estudio de una significativa bibliografía sobre este campo en específico. La aplicación del constructivismo al derecho presenta aportes hermenéuticos de los enfoques doctrinales para establecer un referente bibliográfico. Con este texto el investigador contará con una herramienta de consulta que lo referenciará con el escritor de su incumbencia, evitando un desgaste en el momento de desarrollar su proceso investigativo.

El artículo tiene como objetivo realizar un minucioso sondeo del estado del arte en torno a la aplicación del constructivismo en el campo del derecho, seguido de la discusión hermenéutica de los hallazgos en los escritos que en primera línea fueron objeto de estudio. La metodología utilizada es interpretativa hermenéutica que permitió hacer un rastreo documental amplio y suficiente del estado del arte en cuanto se trata del constructivismo aplicado a la ciencia del derecho.

\section{Estado del arte en torno al constructivismo aplicado al campo del derecho}

En este aparte se hará una revisión de los diferentes tipos de textos significativos que sobre el tema vale la pena traer a colación como son libros, publicaciones, artículos, ensayos y papers, pues así 
será posible mostrar, de acuerdo con los autores, sus posiciones y nuestra intervención hermenéutica a sus proposiciones un aporte bibliográfico que permita a otros investigadores obtener una fuente de consulta amplia, coherente, organizada y lógica. En ese sentido, se desarrollará un repaso por las obras de diferentes pedagogos, filósofos, psicólogos, abogados, docentes y escritores que han analizado, investigado y reflexionado sobre el tema que compete a esta investigación, junto con el análisis y debate a estos criterios desde el punto de vista de la enseñanza del Derecho, los cuales pueden aportar elementos con los cuales construir, con cierta suficiencia, un claro panorama de las investigaciones con evidencia escrita realizadas hasta la fecha en cuanto se trata del constructivismo jurídico.

Es así como finalmente, con este trabajo investigativo de las diferentes teorías con evidencia escrita del constructivismo aplicado al derecho se puede establecer un referente bibliográfico para este caso en concreto.

Duncan Kennedy (1977), por otra parte, además de ser conocido por sus textos críticos sobre decisión judicial ofreciendo una visión fenomenológica de la justicia, también se ha encargado de realizar una crítica directa a la intersección de los derechos y el estudio del derecho critico en general. Dicha crítica se dará en tres partes; una narración del papel que tienen los derechos en la conciencia jurídica norteamericana, una narración sobre cómo paulatinamente podría llegar a perder la fe en la coherencia del discurso de los derechos, por último, una breve indicación del porqué uno podría desear formular tal crítica a pesar de que esto resulte poco agradable. "fenomenológicos" utilizando técnicas empíricas y naturalistas, como la observación participante y no participante para la obtención de datos empíricos como los que suceden en este escenario, en el aula ya sea como "espacio físico o espacio conceptual" (Vasco, 1994, p.57).

Méndez (2014) presenta su tesis, bastante enfocada y precisa, hacia el contenido ideológico de la educación jurídica, a pesar de su notorio carácter aséptico anuncia de manera expresa, para contrarrestarlo, su propósito de politizar el aula. Para lograr formar en las facultades de derecho a este tipo de jurista se debe considerar la importancia del diseño del plan de estudios, en específico, del primer curso, ya que este, a su parecer, puede ser determinante en la formación mental que tendrá el futuro jurista.

Basándose en el pensamiento de Duncan Kennedy, afirma que es perfectamente consciente de los riesgos que conlleva su postura radical, como gusta en calificarla y de las previsibles críticas que puede generar. El riesgo más evidente es que se le pueda acusar de la politización del aula, no como metodología, sino como puro adoctrinamiento político sin más. Esto es algo que como él mismo señala de forma reiterada no sería moralmente aceptable. Politizar sin adoctrinar sería su propuesta, tratando con ello de salvar los inconvenientes sobre todo morales que puede acarrear una práctica docente concebida de este modo.

Guastavino (2011) expone, desde el característico enfoque de la disciplina de Derecho del Trabajo, el conjunto de competencias, genéricas y definidas, entrelaza de una forma directa con todas las habilidades implícitas para la empleabilidad, concepto largamente conocido y conectado, precisamente en el mundo laboral. Siendo de vital importancia el aprendizaje constructivista a lo largo de toda la vida teniendo en cuenta los mercados habituales de trabajo que constriñen a una economía emprendedora y a una mayor afluencia en el empleo, siendo centro de estudio de los modernos iuslaboralistas. 
Nogueira Guastavino (2011) fundamenta el constructivismo como base teórica del nuevo método de enseñanza y el proyecto que desarrolló con el fin de proyectar el Derecho Laboral para enfrentarse con Ios retos que pueden surgir en el siglo XXI. En su reconocida obra denota como del mercado laboral existente en Europa, se deben confrontar retos de conciliación de una manera más laxa, hablando de ámbitos netamente empresariales, supliendo así la necesidad de maximizar la seguridad colectiva, instaurando los tres pilares sobre los que basa la "flexiguridad" (flexibilidad en la contratación y extinción; alta protección social para los desempleados; y activa política de formación y reinserción laboral).

De este modo, se establecen las prioridades y la necesidad de modulares medidas de enseñanza y desarrollo formativo continuo tanto para empleados como para desempleados. Así pues, resalta la economía actual de la clase obrera, en la cual los trabajadores deben estar abiertos a los constantes cambios de su formación para irse acoplando a la demanda del mercado. Se trata, entonces, de institucionalizar "puentes" y conjuntos compensatorios de bienestar, seguridad social que den pie a la activación del empleo y que provean las situaciones dentro del desarrollo de empleo.

De esta manera explica entonces que la empleabilidad debe mantenerse como un mecanismo constante de aptitudes y cualificaciones transferibles que vigorizan el emprendimiento y las capacidades de cada persona, con el propósito de aprovechar las ocasiones de educación y de formación que se les puedan presentar, encaminándose así a la búsqueda de un trabajo decente y de calidad que les permita, bien sea avanzar dentro de la empresa o progresar fuera de ella buscando otro empleo. Esto demuestra que las nuevas competencias y el nuevo método de enseñanza, trascienden a algo más profundo, que un mero capricho académico.

Chamorro (2008), de otra parte un concepto solo adquirirá un estatuto generalizable si está integrado en una red de conceptos que fueron adquiridos con anterioridad en el constructo del individuo; es de vital importancia que el futuro profesional comprenda que un conocimiento solo puede adquirir importancia significativa si, previamente, los elementos que definen dicho concepto han sido abordados en contextos particulares entorno a conocimientos que se han adquirido a través de la experiencia, de ahí que las actividades sueltas, por muy atractivas que puedan parecer, estén condenadas al fracaso dentro de las sendas del conocimiento.

Villa (1999) manifiesta que el constructivismo post-positivista se desarrolla dentro de una disposición que trata puramente de la filosofía analítica, acompaña la epistemología de orientación analítica de la condición que se implanta de forma fija en esta práctica investigativa, al respecto Córtez, Vera, Romero y Chávez afirman:

Especialmente, esta orientación hace parte representativa de las fases históricas de la reflexión epistemológica de la filosofía analítica luego de la etapa crítica del neopositivismo, esta se dio hacia finales de los años 60, fue de este modo que se sumaron tendencias relativistas como las de Kuhn y Feyerabend; en este punto se genera una reacción encaminada al realismo científico. (Cortez, A., Vera, V., Romero, O. y Chávez, J, 2010, pp.105 - 112)

Evans (2004), enfoca su idea en la evaluación continua del estilo de enseñanza del docente y su repercusión en los estudiantes, esto a partir de tres dimensiones la social, el control y la gestión en el aula, describiendo diferentes estilos de enseñanza dentro del proyecto del aprendizaje significativo (Ausubel), con el fin de significar la incidencia de los métodos en la elucubración de ideas propias y la construcción de pensamiento argumentativo. 
Ballester (2002), señala que se habla de aprendizaje significativo cuando el sujeto de aprendizaje hace suyo un nuevo concepto a partir de la relación que establece entre él, es decir, su relación con el objeto de estudio, los conceptos previos y el nuevo concepto, otorgándole un significado en su vida, dando así un carácter funcional- social a lo aprendido.

Cortez, A., Vera, V., Romero, O. y Chávez, J. (2010) afirman que el Aprendizaje Basado en Proyectos (ABP) es una de las formas de trabajo en la que la enseñanza se convierte en un método más positivo que aquellos tipos de educación convencionales, yuxtapuesto en los intereses de los alumnos. De otra parte, para los docentes el desafío de abordar los intereses de los estudiantes implica centrarse en el desarrollo de los proyectos y asegurarse que los alumnos cumplan los requerimientos objetivos que determina el plan de estudio, de manera diferenciada buscando que el proyecto concluya con una evaluación significativa de los avances individuales y grupales, con el debido sustento investigativo. Los proyectos cumplen un papel importante en el aprendizaje y tienen muchas ventajas sobre otras formas de enseñanza en el aula, como las conferencias o los debates.

El constructivismo se enfrentará con dos desafíos, uno, el realismo, el otro, el relativismo, al pretender resolverlos tratará de mantenerse en un camino intermedio entre las alternativas representadas por el realismo y el anti-realismo, el objetivismo y el relativismo; de esta manera intenta no recorrer campos ya transitados, hablando de límites netamente investigativos, así también supone que la ampliación del campo de discusión, no está limitado por el conocimiento científico, sino que se extiende también a otras formas de conocimiento, por ejemplo, al conocimiento de sentido común. A partir de mediados de los años 70 el constructivismo post-positivista se empezará a desarrollar con formulaciones más atrayentes, será fundamentado por Putnam, Goodman, Elgin, Toulmin, Mary Hesse.

Duncan, Kennedy (2015) centraliza la idea de la fenomenología y la crítica de la enseñanza del derecho y la aplicación de las leyes como objeto central de investigación, queriendo producir un istmo que determine la diferencia entre el derecho como fuente creadora de legislación y el derecho como fuente inspiradora de sociedad, introduciendo cambios en la investigación jurídica expresados a través de la crítica socioeconómica y sociocultural, propendiendo por la evolución del sentido trascendental de la organización política y las normas como fuente de ordenamiento del conglomerado.

Erickson (1997) asume como ejemplo el caso de la ejecución de Juana de Arco y cómo el hecho de encontrar el sentido de la interpretación puede considerarse fundamentalmente constitutivo de la vida social humana. Es así como la investigación interpretativa sostiene que la explicación causal en el desarrollo del significado de vida social humana no puede basarse exclusivamente en las similitudes observadas entre conductas anteriores y subsecuentes, incluso si la correlación entre esas conductas parece ser muy fuerte. Por el contrario, una explicación de la causa de una acción humana debe incluir la identificación de la interpretación del significado del actor. El análisis objetivo del significado subjetivo es, por consiguiente, la necesidad intrínseca de las valoraciones al momento de identificar los fenómenos que afectan las masas sociales.

Equipo Uniderecho (2009) el Derecho Penal es la rama del derecho que delimita de manera objetiva la descripción de los delitos, las penas y las medidas de seguridad, siendo su objetivo primordial la conservación del orden social. Desarrollándose como un eficaz método de control social, observando fuertes determinaciones como restringir derechos fundamentales. Es a la vez la descripción clara y evolucionada de la venganza primitiva como sistema de justicia, la cual creaba al final mayores sentimientos sociales de venganza. Ahora es la sociedad organizada política, jurídica y civilmente, 
la que cuenta con los medios legítimos para decir qué conductas serán sancionadas y designar a las personas encargadas de administrar justicia. "Esa discusión muestra que la condición para un discurso intercultural sobre los derechos humanos radica en el propio examen de los discursos intraculturales". (Forst, 2005, p. 26).

Brito Ruiz, (2010), investigó que la justicia restaurativa se centra en la reparación y no en el castigo, es una nueva visión en el tratamiento del delito, Donde la víctima reivindica su papel dentro de la comunidad, y el delincuente entiende las secuelas de su acción, asume su responsabilidad objetiva en el delito y se compromete a reparar los daños. Se trata de una justicia restaurativa que abona a la pacificación social y dignifica la recomposición del tejido social.

Cáceres (2001) describió en su tesis las siguientes elucubraciones: es fundamental aseverar que el derecho es una especie de ingeniería comportamental de la cual surge una realidad social y que a pesar de su vital importancia es una variable más de las existentes dentro del proceso de incitar a la realidad correcta. Lo anterior obligará a los juristas a dejar de lado la hermenéutica y completar los conocimientos con otros métodos, por ejemplo, la ingeniería de procesos, la informática, la sociología y la psicología. Así mismo, ponderar que el desapego del derecho a los textos, solo logra viveza cuando se incorpora a la realidad representativa del mundo y en los comportamientos y situaciones donde las interacciones comunicativas son la base en las que se configura nuestra realidad social, ya que la prioridad de nuestras instituciones públicas es engendrar de manera intensiva estados de cosas que impactan de manera significativa en la formación de la realidad social, será entonces la realidad social global una caja de resonancia.

González (2012) afirma que el resultado más importante es la crítica radical a las suposiciones realistas y descriptivistas, que están presentes de manera relevante en el pensamiento jurídico contemporáneo, sea analítico o no lo sea. Esto también se aplica en gran parte a las manifestaciones de actitudes teóricas de tipo relativista o convencionalista como es el caso del realismo jurídico y la escuela crítica del Derecho; específicamente en casos se mantiene muy fija la idea de que el conocimiento es retrato o representación devota de la realidad, pero hay que tener en cuenta que esta idea no es aplicable en el campo de la experiencia jurídica, este es un terreno adecuado para un amplio conjunto de ordenamientos radicalmente incomparables que los juristas denotarán como actividades científicas, de esta manera, se da el nuevo derecho, intervenciones políticas, etc.

Este sería un inicio del desglose de la teoría que trata de suministrar una paráfrasis constructivista no solo de parte del conocimiento jurídico, sino también del fin que tiene el derecho, así en una concepción de esta clase no es posible separar totalmente el ámbito del método del ámbito objetivo. De este modo, una concreta manera de teorizar sobre el Derecho está regida por una serie de compendios que trabajan como lazos para las iniciativas teóricas viables, también el método elegido interviene sobre la cualidad de configuración del objeto. Así se hace inminente la pretensión de que la teoría jurídica de inspiración analítica que aborde de forma prudente los supuestos filosóficos, epistemológicos, semánticos, que han guiado su característico modo de conducir el análisis teórico en el campo jurídico.

Gómez y Menares (2014) en su artículo analizan los nuevos modelos complejos que deberían considerarse en los ámbitos de la ciencia jurídica y de su enseñanza. Más concretamente, se expone que los argumentos epistemológicos frecuentes determinados por la ciencia en específico por el constructivismo sean adjudicados como un aporte para las nuevas propuestas responsables, que se 
planteen la formación de futuros abogados, con una mejor comprensión del mundo-jurídico junto al compromiso ético-social.

En cuanto al conocimiento lo ven desde un paradigma positivista y conductista que se refleja ámbito del aprendizaje en el que el objeto siempre estará enfocado lejos del sujeto, así no se puede transformar ni perturbar o ser constituido por quien lo estudia; en sus palabras: "Si el sujeto no estuviera, el objeto sería y estaría de igual modo, lo que justifica contextos clásicos de procesos educativos determinados en esta lógica"(Gómez y Menares,2014, p. 23).

Entonces si, por ejemplo, el objeto a enseñar por parte del docente, lo vemos figurado como el contenido, no va a ser concebido de una manera distinta, como sí lo han hecho otras competencias, como el ejemplo que proponen de la biología donde el estudio partirá del tema inicial que es la célula, o en la física que se ve el mismo caso, pero en la partícula, con ello inferimos que la relación que se da entre el alumno-profesor se fundamentará en la dinámica del objeto de estudio y no en la intervención de alguno de estos sujetos. "Hasta el momento no existe unidad de criterio entre los estudiosos respecto a las ciencias propiamente penales, considerado como fenómeno biológico y social"(Gutiérrez, 2009 pág. 2).

En el desarrollo de la formación de las disciplinas jurídicas y su evolución desde la perspectiva de los abogados que lo practican, deberá esta ser determinada por el mismo contexto cultural y cognoscitivo compendioso que los rodea, consecuencia de esto, es menester que los sujetos desarrollen sus destrezas dinámicas, críticas y creativas, esto dado que los conflictos jurídicos, no deben ser delimitados a los tradicionales lineamientos, hecho, ley y aplicación, puesto que se necesitan racionalidades conocidas y postclásicas y de esta manera favorecer los reducidos adelantos de los procesos conflictivos.

Campanario y Moya (1999) concluyen como los defensores de las alternativas repasadas están de acuerdo al señalar que en la mayoría de estas el principal conflicto se encuentra en las resistencias de los alumnos o profesores. Tanto los docentes como las autoridades manifiestan tendencia a ser conservadores al momento de implementar nuevas propuestas de enseñanza. En algunos países, el obstáculo al progreso en cuanto a "el aprendizaje basado en problemas" ha sido justamente el conservadurismo nombrado y el miedo a la innovación. Pero no se debe ignorar que el docente al momento de implementar alguna de las alternativas de enseñanza planteadas, deberá tener claro su posición como autoridad, ya que se podría malinterpretar como una disminución de esta.

Este método no es tan simple como se ve, ya que los problemas deben ser seleccionados cuidadosamente y secuenciados de forma que se consiga el aprendizaje significativo. Debe entenderse la palabra problema en un sentido ambiguo. Esto permite vislumbrar un poco de influencia del método conservador de aprendizaje del derecho en países anglosajones, fundados en el estudio de casos precisos más que en la memorización de códigos y sistemas completos, este tiene como antecedente remoto las posturas filosóficas de John Dewey. Puede que este modelo carezca de algunos aspectos importantes, de esta manera se obligará al alumno a avanzar por sí solo en la solución de estos vacíos, entonces se hace evidente que gran parte de la responsabilidad del éxito de aprendizaje en este método recae sobre el alumno.

Cañete y de la Torre (2011) infieren que el Aprendizaje Basado en Problemas (ABP) es un procedimiento dinámico en el que el alumno cimienta su discernimiento con soporte en un problema 
que es anticipadamente preparado, se presenta con el fin de lograr ciertos objetivos específicos de enseñanza. Para solucionar dicho problema el alumno deberá trabajar competencias delimitadas y a la vez adquirir conocimientos concretos por asignatura.

"Encontramos puntuaciones similares de estrategias, enfoques y actitudes en el pre-test entre los cuatro grupos y diferencias importantes en el pos-test, favoreciendo especialmente a los estudiantes del primer grupo constructivista y con habilidades docentes, que tenían mejores puntuaciones en estrategias, enfoques y actitudes (Gargallo, 2008, pp.425-446). Esta herramienta metodológica exhibe varias ventajas importantes frente a las metodologías conservadoras, toda vez que el alumno en este caso tendrá un postura mucho más activa y dinámica al momento de adquirir los conocimientos, debiendo este asumir responsabilidades, buscando información por su cuenta y organizándola para facilitar su propio aprendizaje, consecuente a esto deberá adquirir una posición reflexiva y crítica. De esta manera se evita que sea solo una simple memorización de información. De otra parte, el trabajo en grupo le permite fomentar el intercambio de ideas y un aprendizaje colaborativo, adquiriendo habilidades vinculadas a la resolución de conflictos y toma de decisiones.

Schmidt (1995) asevera que dentro de la dinámica interna de esta estrategia se anima el aprendizaje autorregulado, es decir, que, durante el análisis inicial del problema, el alumno debe crear un modelo mental referente a la situación que se describe en el enunciado, es decir, que basado en sus propios conocimientos anteriores, crear la puerta de avance hacia la construcción de un nuevo conocimiento, que se produce a través de sus experiencias anteriores a la formulación del ABP.

Monroy (1999) en su tesis pondera como conclusión general que se puede adoptar la afirmación del profesor Matthías Herdegen, quien sustenta que concierne a la formación jurídica transmitir los fundamentos del derecho natural a través de las vías del derecho positivo, este debe orientarse en el sentido de la dignidad humana y los principios de Estado Social de Derecho. El derecho positivo no es solamente un sistema normativo que hace uso de mecanismos para su imposición, sino que comparte con el derecho natural una amplia serie de aspiraciones de bienestar común.

La reforma de la enseñanza del derecho es fundamental para que se pueda cumplir con una verdadera función social y sea un instrumento eficaz en la realización de la justicia. Se hace necesario regular los requisitos básicos de creación y funcionamiento de los estudios de derecho, conviniendo ser flexible para respetar la autonomía universitaria. Do otra parte, se deberá tener vigilancia estricta de parte de las autoridades de gobierno para que siempre se haga efectivo el cumplimiento de dichos requisitos.

Mestre (2018) indica cómo la educación jurídica clínica se ha extendido en los últimos años, siendo muchas las facultades de derecho españolas que acogen, desarrollan o cuentan con algún proyecto de este tipo, que concentra aprendizaje, servicio y transformación, tanto de la enseñanza del mismo derecho como de la sociedad en la que dicho trabajo deberá llevarse a cabo. En las clínicas se aprende derecho en contexto, en acción; se cuentan historias de vida, se identifican estructuras, mecanismos y sistemas de dominio que pasan desapercibidas en las aulas, donde se imparte docencia regular de grado, en este tipo de enseñanza es notorio que lo que se aprende en la práctica es más significativo que aquello que solo se determina por la educación tradicional.

Ausubel (2002) plantea que el aprendizaje del alumno depende de la estructura cognitiva previa que se relaciona con la nueva información, debe entenderse por "estructura cognitiva", al conjunto de conceptos, ideas que un individuo posee en un determinado campo del conocimiento, así como 
su organización. La característica más importante del aprendizaje significativo es que produce una interacción entre los conocimientos más relevantes de la estructura cognitiva y las nuevas informaciones (no es una simple asociación), de tal modo que éstas adquieren un significado y son integradas a la estructura cognitiva de manera no arbitraria y sustancial, favoreciendo la diferenciación, evolución y estabilidad de los subsunsores preexistentes y consecuentemente de toda la estructura cognitiva.

Cuando se está frente al educando dentro de la enseñanza concreta del derecho es procedente determinar sobre diferentes hipótesis que se han diseñado, la teoría del aprendizaje significativo, aprendizaje a largo plazo, o teoría constructivista, según la cual, para aprender es necesario relacionar los nuevos aprendizajes a partir de las ideas previas del alumnado, el aprendizaje para que pueda ser significativo, ha de adquirir la propiedad de ser a largo plazo.

Ribas (2012) precisa que es necesario la pluralidad de los sujetos para la formación de quienes en la sociedad democrática ejercerán la administración de justicia, de ahí se partirá para saber el desarrollo y el resultado del correcto funcionamiento de esta. Observando que nos encontramos en una sociedad donde se incrementa a grandes pasos el nivel de desconfianza, las figuras de autoridad como lo es en este caso el juez, se fuerza que sea necesaria la optimización de la formación, pero la verdadera pregunta sería: ¿Cuál debería ser la formación adecuada para un juez en el ejercicio de su función jurisdiccional? Así las cosas, se hace necesario definir cuál, y cómo será su perfil profesional e indagar las habilidades y competencias, de esta manera se dará el desarrollo metodológico y se logrará el objetivo.

Es inevitable notar cómo el desarrollo del aprendizaje y estudio del derecho, la transformación que este ha tenido está dando un vuelco importante a la sociedad actual democrática y será esto lo que motive una nueva visión sobre la formación dada a nuestros jueces, fiscales y todo el personal encargado de administrar justicia. A lo anterior será entonces factible vincular las nuevas propuestas metodológicas de aprendizaje con los contextos sociales y hacer efectiva la toma de decisiones. Así que lo que se pretende es indagar sobre los aportes que se logran cuando el aprendizaje se basa en problemas y tomar esto como la nueva propuesta educativa y metodológica, propiciando desarrollo de las habilidades de pensamiento que será parte principal en la toma de decisiones del juez; esto nos dará la ventaja sobre otras metodologías, ya que se logra la integración de las habilidades.

Así que al momento de evaluar se deberá tener en cuenta dos criterios relevantes que serán entonces los netamente cognitivos y los integrales que estarán compuestos por la parte social, personal y comunicativa. Estos últimos fácilmente pueden ser evaluados mediante el seguimiento y observación, sin embargo, los cognitivos necesitaran de una demostración práctica y experimental por parte del estudiante. Un ejemplo claro que expone el autor:

Ribas (2012) si pedimos a un alumno que reescriba un texto (de un auto o de una sentencia) redactado de manera errónea o imprecisa, tendríamos que haber definido previamente la reescritura de textos como objetivo del curso, el alumno debería detectar los errores y reescribir el documento correctamente. Esto nos permitirá avanzar a grandes pasos, ya que será posible que sin tener que hacer preguntas se manifiesten las capacidades que el estudiante ha desarrollado, eso sí teniendo en cuenta que es importante tener claros y establecidos los factores a evaluar. 
Rué, Font y Cebrián (2010) afirman que en las valoraciones y evaluaciones hechas a los estudiantes nunca se hace énfasis explícito a la autoevaluación de los aprendizajes, aunque se crea algo innecesario es un aspecto fundamental en el desarrollo del conocimiento, ya que los estudiantes en retrospectiva, apenas perciben y valoran este aspecto de la autorregulación como vinculado a la evaluación. Si se logra una buena autocorrección se alcanzará la cumbre de la metodología de enseñanza, puesto que durante el curso los estudiantes debían proceder a realizar autoevaluaciones del aprendizaje que documentaran por escrito. Los factores culturales siempre tienden a equilibrar la autoevaluación con exámenes escritos, esto denota la insignificancia que se le otorga al método autoevaluativo. La valoración del aprendizaje de ABP es sin lugar a dudas altamente enriquecedora, ya que los estudiantes de derecho han afirmado su empatía por la metodología utilizada y la actuación tutorial.

Olmedo (2011) investigó que pese a los múltiples problemas que presenta la incorporación de este sistema, lo importante es lograr que el alumno vea los resultados que a largo y corto plazo se obtendrán mediante su seguimiento, estos serán superiores a los que se pueden obtener mediante el método tradicional. Una vez se logre demostrar al alumno los beneficios educativos que tiene este, se sentirá atraído por el reto de autosuperación propuesto, de esta forma, se exige a sí mismo mediante la disciplina y estricta dedicación. Por otra parte, el docente luego de superar la mayor parte de problemas circunstanciales que pueden presentarse en el inicio, encontrará la satisfacción cuando el alumno llegue a la cúspide de su aprendizaje, pues esta no será una simple memorización de contenido, sino que será el resultado de un desarrollo práctico.

Forero-Medina y Sandoval (2018) aseveran que al ser la ley una construcción socio-discursiva, siempre nacerá del leguaje aparentemente científico del estudio del derecho que estará a cargo de los profesionales, quienes avalarán las facultades de leyes, así mismo, el bien llamado discurso político se encuentra fundamentado en las dinámicas del poder político y económico que por su naturaleza afectan en las pedagogías educativas hegemónicas.

Silva (2009) recomienda que todo lo anterior conlleva a desarrollar la necesidad de fomentar la obligatoria enseñanza del derecho, así como del procedimiento y todas las posibles habilidades que se han mencionado en la presente propuesta, toda vez que de la experiencia obtenida en la aplicación de los métodos y estrategias para la materia de obligaciones, se pudo observar una serie de beneficios que redundan en la formación de abogados mejor preparados para la práctica profesional. Sin embargo, es necesario matizar que no se trata de fórmulas acabadas y que la aplicación de estos sistemas y destrezas, aunque han producido resultados favorables en la formación de los estudiantes de derecho y pueden ser utilizadas como guías efectivas por los maestros comprometidos con la calidad educativa, lo ideal es que cada docente tomando en cuenta el contenido de su programa, las adecue y pueda complementarlas de acuerdo con sus requerimientos.

La comprobación de la efectividad del método también podrá darse con el seguimiento que se le puede dar al egresado, porque este permite dar un vistazo a los resultados dados. Ya que se observará el uso de todos los conocimientos adquiridos durante la aplicación y la efectividad de los mismos, en el desarrollo socio cultural de la profesión.

González y Muñoz (2010) en sus competencias describen cómo la formación del estudiante de derecho va a estar enfocada al mercado laboral, mientras se pretende que el alumno durante el curso de sus estudios jurídicos, comprenda y desarrolle el conjunto de competencias que le serán 
necesarias para el aprendizaje continuo a lo largo de todo lo que será su labor profesional, teniendo en cuenta que cada vez deberá ser más competitivo para desarrollar un conjunto de competencias, aptitudes y capacidades necesarias para aprender de un modo continuado, pues esto le servirá para desenvolverse en un entorno cada vez más competitivo.

Es importante resaltar cómo el Espacio Europeo para la Educación Superior (EEES) afectará a la docencia universitaria en las facultades de derecho, ya que se verán consecuencias relevantes, una de ellas será la reducción del tiempo presencial de docencia, puesto que añadirá importancia al proceso de aprendizaje del estudiante, donde este será quien incurra en el desarrollo de las investigación, la presencia del docente será necesaria más como un apoyo adicional; sin embargo, esto significa una mayor carga para el docente, quien deberá estar al tanto de los avances de cada alumno y el correcto desarrollo de cualquier corrección posible dentro de todo el proceso, es decir, que aunque se limite el tiempo presencial de la docencia, dedicado a los conceptos básicos y teóricos, se tendrá que dar atención especializada a cada alumno.

Cañas (2015) nos informa el papel decisivo que juega el concepto de competencias, no solo en el campo empresarial, sino también en el educativo, laboral, profesional y todos los necesarios para el proceso de la vida del hombre. El concepto de competencia lingüística se trata entonces de la capacidad de comprensión y producción de nuevas oraciones. Para ser más concisos, la capacidad gramatical según el autor es aquella donde la competencia se materializa en la dialéctica - sociedad - individuo concebidas dentro de experiencias, roles, intercambios; situaciones diversas y complejas, donde el desarrollo de cada sociedad y cultura impone el uso y significado del lenguaje particular.

Fach (2012) anuncia, en primer lugar, las mismas características del Aprendizaje Basado en Proyectos ABP, estas solicitan que a cada docente se le asigne un número reducido de estudiantes, puesto que una de las funciones principales del ABP es usufructuarse de las ventajas y beneficios de la enseñanza especializada, como lo es poder atender el problema multidisciplinar del que se derivan varios factores como lo son los intereses jurídicos del caso, además de la amplitud de conocimientos que los estudiantes van a retener con más facilidad y durante más tiempo, teniendo en cuenta que no solo estará vinculado a un contexto físico, sino también a un contexto fáctico que ha sido generado paulatinamente por los estudiantes. Así las clases se separarán entre las magistrales, ya acostumbradas, y las prácticas del nuevo método, que garantizarán la mezcla natural entre la teoría y la práctica. Así el ABP reduce la brecha que existe entre las facultades de derecho y el ejercicio de la profesión.

Por otra parte, en cuanto al derecho internacional, el ABP ayuda a formar lo que el autor denomina como "licenciados transnacionales", es decir, profesionales que estarán preparados para afrontar exitosamente problemas jurídicos de magnitudes internacionales, desde su país de origen. Para garantizar el éxito del ABP se deberán tener en cuenta factores como, seleccionar un buen problema, garantizar el ejercicio en un grupo reducido de alumnos, un docente que esté formado tanto conceptual como metodológicamente y una planeación previa de cada una de las fases.

Souto (2013) arguye, en definitiva, que educar no se trata solamente de formar, llenando al estúdiate como si fuera un barril, procurando exclusivamente la memorización de la información, evitando la formulación de dudas y la solución de las mismas, por el contrario, se trata de fomentar la creación y solución de dudas, que podrán ser resueltas en la marcha de la práctica y demostrar cómo hacerlo, proporcionando la actitud crítica ante el derecho positivo y la argumentación fundamentada, de tal 
manera que al egresar de la Universidad el estudiante pueda enfrentarse con seguridad a todos los desafíos, incluyendo todos los estudios que deberá asumir a lo largo de su carrera, ya que es de bien saber que la práctica del derecho está en constante cambio, de igual forma, que el alumno pueda dar mejoras al sistema jurídico.

Perilla (2017) advierte que el constructivismo antiformalista atañe de manera dinámica y aplicable todos los instrumentos pedagógicos que puedan ser útiles dentro de la formación jurídica, sujetos a fomentar una innovación de la enseñanza y aprendizaje del derecho. Estos deberán garantizar la participación activa de los alumnos, así como del docente, para que así tengan el estatus social de conocimiento y tenga utilidad a los constructos cognoscitivos. La forma de aplicabilidad será por medio de la iusteoria antiformalista, pues esta comprende el derecho de naturaleza abierta, es decir, será complementado por indeterminadas fuentes a través de las interpretaciones auténticas, se darán en el desarrollo metodológico práctico. Ahora, para poder materializar dichos procesos de desarrollo y crecimiento práctico se deberá abordar el Aprendizaje basado en problemas (ABP).

Vizcarro y Juárez (2008) relatan cómo en una población de Hamilton se da una discrepancia sobre la ineficiencia de los médicos locales a la hora de enfrentarse a problemas de salud. Se constató que estos tenían buenas bases y conocimientos, sin embargo, eran incapaces de dar solución eficiente a los problemas por su falta de experiencia en el campo y su casi nulo desarrollo cognitivo.

David Ausubel, Josef Novak y Hellen Hanesian, (1978) especialistas en psicología educativa de la Universidad de Cornell, que teniendo como precedente a Vygotsky, han diseñado la teoría del aprendizaje significativo, aprendizaje a largo plazo, o teoría constructivista, según la cual para aprender es necesario relacionar los nuevos aprendizajes a partir de las ideas previas del alumnado.

Es factible analizar con argumentos y criterio lógico, luego de una investigación sobre los documentos ya escritos y ratificados en el tema del constructivismo como estrategia pedagógica para la enseñanza del derecho, posteriormente a un barrido y consulta por los diferentes autores y sus escritos vale la pena afrontar la tarea de realizar un aporte al tema en algunas páginas que determinen la necesidad de involucrar el saber del derecho, el constructivismo y la enseñanza basada en problemas como fuente de una ciencia social que debe aportar al Estado y a la sociedad al general un vértice que le permita buscar la igualdad cultural y social a partir de la aplicación de las normas que regulan las relaciones entre el conglomerado social, vamos a partir de diferentes ámbitos del derecho aplicado para tener una idea clara de la necesidad del cambio inminente en la enseñanza de esta ciencia social.

Puceiro (1976) afirma la importancia de descartar la dogmática, como protección en el ámbito del derecho dentro del nuevo ideal científico. Dentro de la ciencia política se ha ejecutado el tema específicamente encaminado a la vinculación que resguarda con la determinada estructura histórica, donde esta nota servirá para la sistematización y organización, desde una orientación relevante de tendencias o corrientes ideológicas. Creando así una articulación teórica donde los sistemas históricos reales dejan de ser el punto focal de la individualización, por el contrario, lo integra como parte específica de la estructura abstracta.

La dogmática dentro del campo científico-jurídico hace referencia a un resultado específico donde los aspectos de orden cultural, científicos y políticos deben ser exactos, ya que estos proporcionan el sentido filosófico en el que se apoyan, las pretensiones del jurista dogmático, deben aportar a un saber de normas de naturaleza trascendental, sociopolíticas junto con los condicionamientos 
ideológicos, cuyas limitaciones deberán ser verificables históricamente hablando. Debe entonces, darse también una identificación sucesiva del derecho como ley y luego como concepto científico y su ingreso al marco sistemático de la teoría.

Teubner (2002) asegura que después de indagar sobre la conciencia colectiva Emilie Dukheim termina donde todo inicia, en el individualismo, de ahí que el miembro individual de la institución piense que su pensamiento está concurrido por el contexto internacional. Ahora dentro de las instalaciones como el derecho, el mismo será quien articule automáticamente la información, fijando objetivos, produciendo construcciones de realidad y definiendo la normatividad. Todo esto surgirá independiente de las construcciones ya hechas en las mentes de los juristas.

Como lo afirma el autor: "El derecho es comunicación y nada más que comunicación". (Teubner, 2002, p.13) a partir de esta afirmación se indaga que será posible evitar los obstáculos del individualismo metodológico, que juzgan al derecho como un conjunto de normas que restringen las acciones individuales. El derecho se explica como un sistema social autopoiético, es decir, un conjunto de operaciones elementales que necesariamente se reproducen. Dicho carácter autoreproductivo no se compondrá ni por normas, ni por legisladores, sino por comunicaciones jurídicas, participación, información y comprensión. Los mensajes jurídicos no pueden acceder al mundo real externo, solo pueden comunicar lo referente a la naturalidad social, es decir, que el Derecho reinventa la realidad social, sin embargo, la perspectiva constructivista no se debe relacionar con el solipsismo metodológico, más bien busca un punto de equilibrio entre estos dos. El sistema comunicativo del derecho no es susceptible a los procesos psíquicos de los juristas y viceversa: el derecho no tiene acceso a los procesos psíquicos. Las personas son indispensables para la comunicación jurídica, ya que el derecho, como proceso social, necesita atribuir la comunicación a un actor individual con el fin de proseguir con su autorreproducción.

Lovece (2001) explica como el derecho interpretado desde el punto de vista epistemológico como herramienta primordial en la construcción de un Estado Social de Derecho es un paragón sin medida, procurando entregar en gran medida a la administración de justicia la responsabilidad de interpretar las leyes emitidas por el poder legislativo, con el objeto claro y preciso de evolucionar junto a la aplicación de la norma a la sociedad que presume su propuesta como un acto de regulación expresa de los fenómenos socioculturales que atañen al Estado en sí.

Pulido (2013) insiste en tener el transicional enfoque dogmático de la enseñanza y el aprendizaje, sea por las razones de miedo o deslealtad al formato tradicional. Sin embargo, es claro que dicho enfoque tradicional falla al no lograr comprometer a los estudiantes en la participación activa de su aprendizaje, ya que se expone un énfasis principal en la memorización de los contenidos impartidos en clase. Por lo anterior, el enfoque tradicional incapacita el desarrollo de un pensamiento crítico, que es algo fundamental en un estudiante de derecho. "Se discute el origen y la elaboración del estilo de aprendizaje como concepto, trazando la influencia de una cognición y un enfoque centrado en el aprendizaje de la psicología de la diferencia individual”.(Rayner y Riding, 1997, pp. 5-27)

Vasco (1990) concibió la filosofía educativa como manera particular de desarrollar la teoría constructivista, orientando al alumno a la autonomía dejando de lado la pregunta básica de la educación de la Edad Media que verificaba la supuesta verdad para poderla impartir en las aulas de clase y paso a ser la evolución filosófica en preguntarse ¿Se puede enseñar? De allí que genere una función básica de la construcción de la enseñanza del Derecho, enfocando al estudiante en su 
continua capacidad de discernir y crear conciencia social, siendo prolijo en determinar su capacidad para el mejoramiento de la aplicación y creación de legislación.

Zaffaroni (1982) afirmó que la antropología filosófica y el derecho, y en particular el derecho penal como saber jurídico, cuentan con diferentes puntos de desacuerdo a lo largo de la historia. La idea de ayudar a la humanidad a regular sus relaciones como entre sí y no como talanquera que obstruya el devenir de las variantes que regulan los comportamientos entre los componentes humanos de la sociedad. Hablando en términos más precisos y claros: humanista nos dice si nos hallamos ante un derecho penal liberal o ante un derecho penal autoritario. El derecho penal es un saber, no son las leyes penales, ajustándose a un sistema de interpretación que hace el jurista. Estas normas pueden ser aberrantes como son las que autorizan la tortura o que prescriben penas crueles y desproporcionadas. La función jurídica del saber no es racionalizar las leyes, sino descubrirlas, denunciarlas y brindar los argumentos para que estos descarten o no su aplicación.

Es de la teoría constructivista afirmar que la experimentación y la participación activa forman parte esencial para la retención del conocimiento, mientras el pensamiento creativo y la motivación vocacional del estudiante se consigue con la interacción entre estos, ya que serán los estudiantes quienes por medio del uso del material desarrollado por ellos mismos, encuentren nuevas dudas que les permitan llegar a conclusiones individuales o grupales.

Osika, Giménez, Benítez, y Álvarez (2008) divulgan que las prácticas pedagógicas construyen conocimiento colectivo en el aula y las mismas ponderan los procesos de reflexión crítica y argumentación, el trabajo asociativo, los focos de debate y hermenéutica, el respeto por el otro. Es así como propendiendo por el uso de estas prácticas el pedagogo fortalece su saber académico, puesto que se ve obligado a reflexionar sobre su práctica, guiando al aula de clase a un proceso continuo de mejoramiento y de insurgencia educativa.

Marx (2004) marca un punto importante donde se resalta el rompimiento teórico y político que existe con la filosofía del derecho hegeliano de izquierda y la filosofía del derecho activista, esta ruptura llevará a definir por primera vez la concepción materialista como así lo define, de la relación entre la teoría y la práctica en la aplicación de las leyes como objeto de apoderamiento del actuar social.

Ahumada (1983) afirma que la educación es una etapa de transición entre el individuo y su identidad social. Por ello, se hace necesario diseñar un modelo integral que se base en la enseñanza comprensiva, integradora, unificada y diversificada a través del aprendizaje significativo, que haga posible ampliar en el educando las posibilidades de una mejor educación, brinde una mejor oportunidad a niños y jóvenes con conductas atípicas y limitadas, descubra sus habilidades, destrezas y capacidades académicas e interpersonales para que a partir del autoaprendizaje puedan desarrollar y ejercer su liderazgo.

María Paula (2004) refrenda que el aprendizaje en colaboración es una práctica pedagógica consistente con el constructivismo que según se demuestra tras varios estudios empíricos, logra que los estudiantes comprendan el contenido del campo que estudian, que manejen el leguaje del mimo, que desarrollen el pensamiento crítico, todo esto siempre guiado bajo la supervisión y apoyo del docente. 
Castaño Ramírez, Velásquez Sánchez, Cardona Agudelo, Blandón Grajales, Castaño López y Marín Noreña (2014) advierten que el aprendizaje problemático, a diferencia del enfoque pedagógico tradicional, el cual se basa en el docente pasivo, memorístico, cuyo fin es la memorización de información por medio de la repetición, aquí los estudiantes se transforman en parte activa y el objetivo del docente será guiar al estudiante en forma abreviada bajo una visión hermenéutica de la historia del conocimiento; para que de esta manera las dificultades presentadas puedan ser superadas como parte del proceso, prestando mayor importancia a la forma cómo lograron aportar nuevas soluciones.

Es ahí donde el estudiante toma potestad sobre la información y empieza a relacionarla con los contenidos previos y transfórmalos en nuevas elucubraciones. Además de esto empieza reconocer las diferencias lógicas existentes con los autores e inicia a darles solución. Esto propicia desarrollos cognitivos y argumentativos, lo que logrará que el alumno desarrolle la capacidad de aprender a aprender. La manera de solucionar problemas será autónoma y espontánea, llevado a un campo cotidiano, es así como el principal objetivo del ABP es lograr que el alumno adquiera la capacidad de plantearse y dar solución a problemas como la forma de adquirir conocimiento.

Romero (2009) expone que el ABP se ha desarrollado, desde sus comienzos, como nueva forma de educación, que se caracteriza porque el aprendizaje está centrado en el estudiante, promoviendo que este sea constructivo y con significado intrahumano, además de desarrollar una serie de habilidades y competencias indispensables en el entorno profesional. De otra parte para el EEES, la universidad debe formar titulados con perfiles que respondan a una demanda activa, más interdisciplinaria y flexible; profesionales que estén en constante regeneración educativa, que permita el desarrollo de los diferentes campos de las ciencias y aporten al continuo mejoramiento de la humanidad como conglomerado social, responsable del futuro del planeta.

Forst (2005) plantea una discusión acerca de la fuente y vigor de los derechos humanos y sustenta que su argumentación puede encubrir presunciones políticas y económicas de Estados con la finalidad de conquistar o conservar su autoridad en un territorio específico. Inicia con estudios de las críticas a la generalidad de los derechos humanos para comprender su base normativa; luego indaga sobre otros inconvenientes implícitos en la teoría de estos derechos, como la analogía entre derechos morales y positivos y la tracción entre derechos humanos y democracia.

Gómez (2014) analiza los nuevos y confusos paradigmas que elementalmente deben ser considerados en los ambientes de la ciencia jurídica y de su instrucción. Específicamente, se formula que los contextos cognoscitivos generales que establecen a la ciencia en especial el constructivismo sean tomados como primordial contribución para las nuevas propuestas responsables, que se planteen en la formación de los que serán futuros abogados, logrando así una comprensión óptima del mundo-jurídico y con compromiso ético.

González Galván (2012) asevera que el aprendizaje jurídico debe ser concordante con la dinámica social. El discernimiento de la inteligencia por medio de la neurociencia nos sitúa en el camino a considerar que la formación debe pertenecer ahora a las variadas inteligencias humanas como los son, intelectual, emocional, intuitiva, social, ecológica, cosmológica. El conductismo jurídico transmitió al profesor el monopolio del saber y este a su vez lo acusó a los estudiantes; el constructivismo jurídico, por su parte, sitúa enfoque en el estudiante como constructor de su propio conocimiento, por lo cual se plantea como síntesis. 
Philippe (2009) afirma que el aprendizaje jurídico debe ser concordante con la dinámica social, argumenta que ambos consientan a la fabricación de ideas trabajando de manera contigua. Este discernimiento resultado del diálogo responsable en las aulas hará que se reproduzca en nuestra sociedad una democracia política.

Gorali (2018) infiere respecto de la crítica que es una reflexión precisa sobre lo que nuestros conocimientos nos impiden comprender. Una manifestación que logra transfigurar nuestro pensamiento a partir de la interrogación misma. Bajo esta premisa su pretensión es la de repensar la relación entre enseñanza, crítica y acción en el campo jurídico, reiterando en la penuria de reinscribir la crítica no como descubrimiento de una única "verdad esencial", sino como una acción que transfigure partiendo de los propios límites que el ordenamiento establece, dando nacimiento a la nueva ciencia. Aborda la pregunta por la exégesis judicial insinuando la necesidad de desplazar el "trabajo jurídico" hacia el ámbito de la "acción". El trabajo o la elaboración se causan en aislamiento, por el contrario, la acción requiere de ajenos.

Hernández (2014) aborda la importancia de la teoría constructivista como una representación afanosa que busca la modificación en la relación con el derecho internacional de los derechos humanos, el Estado y las empresas transnacionales, dicho cambio se debe ver reflejado en la restructuración de las leyes internacionales mediante la invención de normas que los vinculen naturalmente, al igual que la instauración de un orden social internacional que tenga como propósito el amparo de los derechos fundamentales de manera especializada y coadyuvante a la labor doméstica que detentan Ios Estados. Esta falta de perspectiva constructivista acarrea el problema de la efectividad de los derechos humanos en los Estados, especialmente en el colombiano, en un juego en el que las empresas transnacionales sobreponen sus intereses sobre los seres humanos.

Marroquín (2006) afirma que en general la aplicación del enfoque constructivista es escaso, ya que no se explora lo suficiente en ninguna de las áreas. Lo que predomina es el aprendizaje sistemático y tradicional. La recomendación es realizar análisis más específicos sobre este argumento con el apoyo de personas experimentadas en estos temas, para así definir cómo se están aplicando los contenidos factuales, de conceptos concordantes con el fin de poder aplicarlos a cada facultad, con el objetivo de planificar capacitaciones sobre la aplicación de modelos educativos.

Sandoval (2018) reitera que se hace necesario incluir en el aula el cuestionamiento sobre la contradicción jurídica, supuesto fundamental para generar un pensamiento crítico en los juristas modernos, para ello, será necesario delinear un bosquejo general que permita comprender, lo que la aproximación a los estudios críticos legales en sus siglas en ingles CLS es, emprender una investigación conjunta con los trabajos al interior del movimiento CLS sobre la contradicción, particularmente los producidos por Duncan Kennedy, por último, sin dejar de ser importante revisar si en la doctrina europea y latinoamericana esta idea ha tenido repercusión y, de ser así, cómo se ha efectuado su análisis.

Pablo (2013) afirma que la pedagogía universitaria quizá haya sido, en ámbitos jurídicos, la que más tardíamente abandonó la perspectiva instructiva para así descubrir con mayor retraso, las doctrinas constructivistas del aprendizaje. La metodología del Aprendizaje basado en problemas ya aplicado y con eficacia, en otras ramas jurídicas se ensaya con problemas de los que pretende dar cuenta esta comunicación en el Grado en Derecho de la Facultad de Derecho de la Universidad de Salamanca. 
Puceiro (1976) acuña que la tradición iusnaturalista que es la base del constructivismo moderno, es aquí donde recupera la función que se le negará a lo largo de las distintas etapas evolutivas del sistema. El fin general del derecho es ejercer la ley moral del hombre, bajo el punto de vista religioso. El cristianismo no existe solamente como regla de las acciones, sino como fundamento de comportamiento.

Hernández (2014) asevera la importancia de la teoría constructivista como una visión dinámica que se encarga de buscar un avance entre la relación existente del derecho internacional y de los derechos humanos, el Estado y las empresas transnacionales y para que este cambio se vea plasmado en la restructuración de las leyes internacionales por medio de la creación de normas que los vinculen y así se lograra la búsqueda de la protección de los derechos fundamentales. La falta de perspectiva constructivista conlleva a los problemas de la efectividad de los derechos humanos en los Estados y en un juego en el que las empresas transnacionales sobreponen sus intereses sobre los seres humanos.

Cobo (2008) para quién el constructivismo pedagógico ocurre a través de una interacción de la nueva información con las ideas pertinentes que existen en la estructura cognoscitiva. Como ejemplo se puede tomar el área del derecho penal en el caso del hurto (artículo 239 del Código Penal) como se juzga de forma diferenciada a una persona que se apodera de un alimento ajeno por estado de necesidad que a otra que lo hace de forma dolosa saca de la esfera de dominio de su propietario una serie de elementos al ingresar a su sitio de habitación de manera soterrada, finalmente se trata del mismo delito y es costumbre en la justicia penal imponer la misma condena para ambos actores sin tener un criterio subjetivo.

Patiño (2005) analiza la Teoría impura del derecho, de Diego Eduardo López, afirma que esta fue recibida por la comunidad académica de la división Filosofía-Derecho como un magnífico aporte al tradicional cultivo de la filosofía latinoamericana, dando una nueva perspectiva a la crítica al derecho, horizonte propio de los currículos de enseñanza de las facultades.

Rubio (2017) denota cómo el Grado en Relaciones Laborales y Recursos Humanos no tiene un campo de conocimiento específico, más bien se configura de tal manera que engloba conocimientos jurídicos y gestión del personal, es decir, respecto al Derecho procesal, el plan de aprendizaje no tendrá ninguna asignatura introductoria que permita a los alumnos establecer una primera toma de contacto con la terminología y la dinámica de esta disciplina, por este motivo para los alumnos es tan complicado cuando tienen que enfrentarse con materias como el Derecho Procesal. A fin de disminuir estas dificultades se desarrollan diferentes técnicas docentes enfocadas por el modelo de clases participativas, donde tanto los alumnos como los docentes serán participes del aprendizaje.

Tapia Argüello (2018) estudia las relaciones entre el discurso educativo de los derechos humanos y la Crítica jurídica, se enfoca principalmente en lo que puede llamarse la "crítica negativa" al derecho y los derechos. A pesar de la relevancia que tiene la diatriba negativa para todo discurso crítico, en especial para los que estudian algo que juzga estar más allá de las opciones de la sátira, presenta contrariedades que configuran un rechazo a los derechos humanos en las sociedades coetáneas.

Villegas (2013) propone que partiendo de la recuperación de la sociología, el posestructuralismo y la historia de los lenguajes políticos se hace más radical la crítica jurídica logrando un distanciamiento de cualquier tratamiento sistémico de lo social y el derecho. El fin es una actitud radicalmente 
crítica respecto de lo jurídico y tendrá dos puntos centrales, uno es el cuestionamiento constante de fundamentos del derecho aceptados por la doctrina jurídica; el otro es el énfasis en las instancias fundacionales de las estructuras jurídicas de sentido.

Bedoya (2011) tiene como propósito tomar los elementos de la argumentación y la hermenéutica jurídica, como métodos para justificar las decisiones judiciales que han adquirido especial relevancia en el marco de la construcción del derecho en Colombia. Estos métodos basados en principios y valores significan un avance en el establecimiento de una nueva concepción de la validez jurídica.

Comparado estos dos enfoques jurídicos: la argumentación y la hermenéutica, podemos aseverar que, aun teniendo diferencias, ambos resaltan el punto de vista valorativo en el razonamiento jurídico y la supremacía constitucional en la tarea judicial.

\section{Materiales y métodos}

Este artículo corresponde a la metodología con enfoque histórico-hermenéutico y analítico. Dos son los recursos metodológicos mediante los cuales se ha de abordar la investigación: el método histórico hermenéutico que permite abordar la comprensión de los escritos, conceptos e investigaciones adelantadas en el área del constructivismo enfocado en la enseñanza del derecho, tal como fueron comprendidos en el momento de la historia que trata. Partiendo de la concepción hermenéutica que defiende el carácter social e histórico de los conceptos que son dinámicos, cambiantes y construidos de forma social e intersubjetiva, puede abordarse la forma en que ellos se van modificando y extendiendo en el tránsito por la formación escrita e investigativa sobre el constructivismo. Dicha teoría considera, además, que los enfoques investigativos y los conceptos tienen una influencia en la práctica, lugar donde son aplicados y llevados a cabo. De esta manera, los conceptos y los enfoques pedagógicos se convierten en pautas y esquemas para la acción, por lo cual contribuyen, a través de la mediación de imaginarios prácticos, en móviles para la vida experiencial. La hermenéutica argumentativa y crítica ofrece el recurso de la articulación entre la parte y el todo que permite interpretar constantemente los miembros de la red de enfoques formativos en relación con la totalidad del sistema de representaciones del nuevo orden social y político. Así mismo, permite el tránsito de las ideas de los diversos grupos sociales a la comunidad como totalidad. El camino es siempre de ida y vuelta, y enriquece la comprensión de los diversos elementos.

El método analítico es un mecanismo que permite el análisis de los escritos encontrados en el ámbito del constructivismo aplicado a la enseñanza del derecho, entendido en sus relaciones de solidaridad e interdependencia, que constituyen la representación de las metodologías educativas, de manera tal que el análisis da las pautas para la interpretación, que se enriquece con ella. Lo que se pone en juego en últimas es la confrontación de interpretaciones: de la interpretación que hacemos hoy, los diferentes escritos de investigadores del siglo XXI en Colombia, con su consecuente interpretación y la apropiación que hicieron de los conceptos y las teorías algo más cercano a la enseñanza del derecho como área de las ciencias sociales, así como con la interpretación de las consecuencias que tuvieron dichas apropiaciones en las maneras que usamos para formar pedagógicamente hoy en día.

En la investigación se encuentra determinada a utilizar una fuente principal: como lo son los escritos investigativos en sus diferentes versiones libros, artículos, tesis, papers y demás documentos físicos y electrónicos de interés, afianzando la idea del constructivismo como teoría pedagógica aplicable al derecho. Dada la dificultad para presentar en este espacio el marco teórico completo, las obras por 
utilizar serán referidas en la bibliografía.

Entre algunas cuestiones evidenciadas a través de la lectura del horizonte constructivista aplicado al derecho tenemos la comprensión de la influencia en la investigación como totalidad desde la comprensión razonada de las partes en su relación individual y grupal; también tiene en cuenta la influencia de la motivación previa para el posterior proceso de aprendizaje y cómo dicha estimulación puede lograr resultados más evidentes en la comunidad educativa, tanto en docentes como en estudiantes. De esta manera, incluso los conceptos más abstractos pueden ser deducibles y analizables desde una postura pedagógica, teórica, histórica, hermenéutica y analítica. Además, tiene la prerrogativa de poner en discusión y complementar diversas disciplinas y campos del saber cómo son la Filosofía, la Antropología, la Sociología y la Economía.

\section{Conclusiones}

Con relación al problema postulado: ¿Cuáles son las investigaciones más relevantes que se han trabajado en términos del constructivismo aplicado al derecho? de acuerdo con los resultados obtenidos se respondió conforme al desarrollo de los objetivos específicos de la manera siguiente:

Las investigaciones que se han desarrollado en torno al constructivismo aplicado a la enseñanza del derecho y el enfoque constructivista significativo son pertinentes para obtener un marco teórico que sirva a los investigadores en la asignatura de derecho enfocado a la enseñanza constructivista, aunque aún no se ha desarrollado suficientemente la indagación en este aspecto, el artículo servirá como texto guía al momento de investigar, mostrando al estudioso el estado del arte frente al tema y los referentes que pueden servir de marco teórico a su análisis; así como las diferentes posturas en cuanto al constructivismo aplicado al derecho concretando la cátedra enfocada a la solución de problemas como aproximación del constructivismo aplicado al derecho.

En este aspecto, es posible decir que la anticipación de sentido permite deducir que la teoría constructivista aplicada al derecho en su soporte documental fomenta la futura investigación, teniendo un marco conceptual y un estado del arte propicio para indagar con mayor precisión dependiendo del área de estudio en la enseñanza del derecho. Al describir la teoría constructivista y sus contribuciones al aprendizaje para adecuarlas a la enseñanza del derecho penal.

Se relaciona cómo la aplicación del constructivismo y del significado pueden aportar de manera clara y congruente una visión generadora de crítica social, filosófica, psicológica, cultural, atañendo la relación maestro - estudiante - derecho en permanente evolución, otorgándole a las partes las herramientas necesarias para una constante búsqueda de nuevo conocimiento enfocado en la consecución de justicia social y material, en las diferentes etapas de su carrera y en su obligación moral y social de aplicar el derecho como generador de eventos de paz y justicia.

Se promueve una reflexión frente a las lecturas efectuadas como referentes para la consecución del estado del arte, con una visión crítica argumentativa que perfile la idea del investigador, en cuanto corresponde a los textos desarrollados en referencia con la aplicación del constructivismo como método de enseñanza en el ámbito del derecho.

Se genera nuevo conocimiento a partir del estado del arte obtenido y de cómo el aprendizaje significativo en el área de estudio aquí planteada conlleva a nuevos paradigmas del conocimiento en los factores sociológicos, filosóficos, religiosos, políticos y legales para crear una nueva concepción 
de la aplicación subjetiva de la ley. De tal manera, se beneficiarán no solo los docentes, que tendrán a disposición una fuente fidedigna de consulta, así como demás investigadores que ante la reflexión conseguida podrán extraer información fiel y cierta para llevar a cabo sus propios objetivos académicos.

Este artículo aporta un enfoque hermenéutico de los diferentes textos y aportes constructivistas en el derecho estableciendo un referente bibliográfico de interés para la comunidad investigativa. Ejemplificando el ámbito de aplicación de la teoría constructivista y del aprendizaje basado en problemas aterrizando el tema en cuestión con una opinión propia que genere un contexto que delimite la discusión al análisis de las lecturas efectuadas durante la investigación de escritos que se han desarrollado en cuanto se trata del constructivismo aplicado a la enseñanza del derecho, esto permite la estimulación del pensamiento crítico y reflexivo tanto de los contenidos que académicamente se les expone como de los contextos y situaciones que la realidad les impone, como seres sociales, políticos, religiosos y culturales.

En este sentido según las lecturas efectuadas; el constructivismo aplicado a la enseñanza del derecho fomenta el pensamiento crítico y permite la construcción de nuevo conocimiento, fortalece la capacidad de crítica racional- social en los estudiantes de derecho. Se hace indudable la importancia de la investigación, reflexionando pacientemente y a consciencia, realizando un análisis hermenéutico de los textos que se producen luego de la actividad investigativa de los especialistas en la materia y proporcionar la bibliografía de los contenidos para colaborar con el trabajo investigativo, creando un contenido con las características que permiten la consulta fácil de aquellos textos que desarrollan el área del saber que nos compete en el presente documento como lo es el constructivismo aplicado al derecho.

\section{Referencias bibliográficas}

Ahumada, W. (1983). Mapas Conceptuales Como Instrumento para Investigar a Estructura Cognitiva en Física. (Tesis de Maestría). Instituto de Física Universidad Federal de Río Grande Do Sul Sao Paulo.

Ausubel, D., Novak.J y Hanesian, H. (1978). Educational psychology a cognitive view. New York: Holt, Rinehart and Winston. Reapers.

Ausubel, D. (2002). Adquisición y retención del conocimiento. Una perspectiva cognitiva. Buenos Aires: Paidós.

Ballester, A. (2002). El aprendizaje significativo en la práctica, cómo hacer el aprendizaje significativo en el aula. España. Recuperado de http://eduteka.icesi.edu.co/pdfdir/EIAprendizajeSignificativoEnLaPractica.pdf

Brito, D.(2010). Justicia restaurativa. Reflexiones sobre la experiencia de Colombia. Ecuador: Universidad Técnica Particular de Loja.

Chamorro, M. (2008). Didáctica de las matemáticas. Madrid: Pearson Prentice Hall.

Cobo, E. (2008). Una propuesta para el aprendizaje significativo de los Estudiantes de la escuela San José La Salle, de la ciudad de Guayaquil. Ecuador: Universidad Andina Simón Bolívar. 
Cortez, A., Vera, V., Romero, O. y Chávez, J. (2010). Construcción del conocimiento desde un enfoque constructivista con soporte tecnológico. Revista de Investigación de Sistemas e Informática (RISI) $7(1), 105-112$.

Evans, C. (2004). Explorando la relación entre el estilo cognitivo y el estilo de enseñanza. Educational Psychology, 24(4),509-530.

Duncan, K. (1977). Critica de los derechos. Revista Jurídica de la Universidad de Palermo 7 (1) 47-80. Recuperado de https://www.palermo.edu/derecho/publicaciones/pdfs/revista_juridica/n7N1-Julio2006/071Juridica03.pdf

Duncan, K. (2015). Tres globalizaciones del derecho y del pensamiento jurídico, 1850-2000. Bogotá: Universidad Externado de Colombia.

Erickson, F. (1997). Métodos cualitativos de investigación sobre la enseñanza: en la investigación de la enseñanza. Barcelona: Paidós.

Equipo Uniderecho. (2009). El Derecho Penal. Minutas noticias consultorio jurídico. Recuperado de https://www.uniderecho.com/leer_tarea_Derecho-Penal_13_1440.html

Forst, R. (2005). El derecho básico a la justificación: hacia una concepción constructivista de los derechos humanos. Estudios políticos, (26), 27-59.

Gómez, F y Menares Ossandón, N. (2014). Los aportes de paradigmas complejos y constructivistas para la enseñanza de la ciencia jurídica. Ius et Praxis, 20(1), 199-220.

González Galván, J.(2012). El constructivismo pedagógico aplicado al derecho: hacia una formación dinámica. Boletín mexicano de derecho comparado, 45(133), 119-139.228

Gorali, M. (2018). Enseñanza, crítica y acción en el campo jurídico: pensando junto a Duncan Kennedy. Revista Electrónica Instituto de Investigaciones Jurídicas y Sociales AL Gioja, (20), 248-259.

Gargallo, B. (2008). Estilos de docencia y evaluación de los profesores universitarios y su influencia sobre los modos de aprender de sus estudiantes. Revista Española de Pedagogía, 66, 425-446.

Gutiérrez I. (2009). Derecho Penal. Bogotá. Recuperado de https://www.academia.edu/5355951/ Derecho_penal_Irma_Guti\%C3\%A9rrez_\%C3\%81vila

Hernández, A. (2014). El constructivismo en la relación derecho internacional de los derechos humanos, el estado y las empresas transnacionales. (Tesis de grado). Universidad Militar Nueva Granada. Bogotá. Recuperado de https://repository.unimilitar.edu.co/bitstream/handle/10654/11690/ re\%20nuevo\%20trabajo\%281\%29.pdf?sequence=1\&isAllowed=y

Méndez, C. (2014). Kennedy, Duncan, La enseñanza del derecho como forma de acción política, Buenos Aires, Siglo Veintiuno Editores, 2012, 110 pp. Boletín Mexicano de Derecho Comparado, 47(139), 351-355. Recuperado de http://www.scielo.org.mx/pdf/bmdc/v47n139/v47n139a14.pdf 
Marx, K. (2004). Crítica de la filosofía del derecho de Hegel. Buenos Aires: Ediciones del Signo.

Lovece, G. Construcción social del derecho: aproximaciones al debate del siglo XXI. Revista da Faculdade de Direito, 1(20).

Osika, R. Giménez, M. Benítez, M y Álvarez, I. (2008). Los proyectos de investigación en el aula, un nuevo enfoque en la enseñanza de la química. Recuperado de www.unne.edu.ar/Web/cyt/cyt/2001/ cyt.htm

Patiño, A. (2005). Filosofía "impura” latinoamericana y Teoría impura del derecho, de Diego Eduardo López. IUSTA, 22(1), 161-180. DOI 10.15332/25005286

Rayner, S. and Riding, R. J. (1997). Towards a Categorization of Cognitive Styles and Learning Styles, Educational Psychology, vol. 17 (1), 5-27. https://doi.org/10.1080/0144341970170101

Romero, F. (2009). Aprendizaje significativo y constructivismo. Revista digital para profesionales de la enseñanza, 3,1-8. Recuperado de https://www.feandalucia.ccoo.es/docu/p5sd4981.pdf

Sandoval, L. (2018). La "contradicción fundamental" en el movimiento critical legal estudies. Temas Socio-Jurídicos, 37(74), 68-84. Recuperado de https://www.academia.edu/38883405/La_ contradicci\%C3\%B3n_fundamental_en_el_movimiento_critical_legal_studies

Tapia Argüello, S. (2018). The Modern Possibilities of Human Rights. A Critique of the Negative Critique of Law and Rights. Mexican law review, 10(2), 139-154. http://dx.doi.org/10.22201/ iij.24485306e.2018.20.11896

Vasco, C. (1990). Pedagogía, Discurso y Poder. Bogotá: Corprodic.

Vasco, E. (1994). Maestros alumnos y saberes: investigación y docencia en el aula. Bogotá: Cooperativa Editorial Magisterio.

Villa, V. (1999). Constructivismo y teoría del derecho / Vittorio Villa. Doxa: Cuadernos de Filosofía del Derecho, núm. 22, 285-302. DOI http://dx.doi.org/10.14198/DOXA1999.22.11

Werbel y Peck, (1983). Psicología Educativa: Un punto de vista cognoscitivo. México: Trillas

Zaffaroni, E. (1982). Derecho Penal. Parte General. Buenos Aires: Sociedad Anónima Editora, Comercial, Industrial y Financiera. 